\title{
Pangolin v1.0, a conservative 2-D advection model towards large-scale parallel calculation
}

\author{
A. Praga ${ }^{1}$, D. Cariolle ${ }^{1,2}$, and L. Giraud ${ }^{3}$ \\ ${ }^{1}$ Centre Européen de Recherche et de Formation Avancée en Calcul Scientifique/Centre National de la Recherche \\ Scientifique, URA 1875, Sciences de l'Univers au CERFACS, Toulouse, France \\ ${ }^{2}$ Météo France, Toulouse, France \\ ${ }^{3}$ Institut National de Recherche en Informatique et en Automatique, Talence, France
}

Correspondence to: A. Praga (alexis.praga@gmail.com)

Received: 1 July 2014 - Published in Geosci. Model Dev. Discuss.: 18 July 2014

Revised: 12 December 2014 - Accepted: 22 December 2014 - Published: 9 February 2015

\begin{abstract}
To exploit the possibilities of parallel computers, we designed a large-scale bidimensional atmospheric advection model named Pangolin. As the basis for a future chemistry-transport model, a finite-volume approach for advection was chosen to ensure mass preservation and to ease parallelization. To overcome the pole restriction on time steps for a regular latitude-longitude grid, Pangolin uses a quasi-area-preserving reduced latitude-longitude grid. The features of the regular grid are exploited to reduce the memory footprint and enable effective parallel performances. In addition, a custom domain decomposition algorithm is presented. To assess the validity of the advection scheme, its results are compared with state-of-the-art models on algebraic test cases. Finally, parallel performances are shown in terms of strong scaling and confirm the efficient scalability up to a few hundred cores.
\end{abstract}

\section{Introduction}

Global three-dimensional chemistry-transport models (hereafter referred to as CTMs) play an important role in monitoring and predicting the composition of the atmosphere (e.g., Chipperfield, 2006; Teyssèdre et al., 2007; Huijnen et al., 2010). Those models include large-scale transport, emissions and chemical transformations of trace species, and sub-scale grid processes like convection and deposition. In CTMs, advection by large-scale winds is a key process that must be handled by numerical algorithms. For these algorithms, mass conservation for the considered species, monotonicity and numerical accuracy are especially important for long simulations where accumulation of errors and bias must be avoided.

In this paper, we present a conservative advection model on the sphere which is intended to form the basic framework for a future CTM. The adopted scheme is based on a flux-form (Eulerian) tracer advection algorithm on a reduced latitude-longitude grid. A finite-volume approach was chosen as it provides an easy way to ensure mass preservation. Furthermore, parallelizing the model is then reduced to a classical domain decomposition problem.

The specificity of our model, named Pangolin ${ }^{1}$, lies in the grid definition, where the number of cells on a latitude circle is progressively decreased towards the pole in order to obtain a grid which approximately preserves the cell areas at mid- and high latitudes. This avoids the so-called "pole problem" arising from the convergence of the meridians, which severely limits the size of the time steps for Eulerian models. Several approaches have been adopted in previous studies to cope with this issue. Finite-volume schemes on latitudelongitude grids often aggregate latitudinal fluxes near the poles (e.g., Hourdin and Armengaud, 1999) or successively double the grid size at high latitudes (e.g., Belikov et al., 2011).

Alternatively, quasi-uniform spherical grids have been developed, such as a cubed-sphere ${ }^{2}$, composite mesh -

\footnotetext{
${ }^{1}$ PArallel implementatioN of a larGe scale multi-dimensiOnaL chemIstry-traNsport scheme

${ }^{2}$ Each face uses Cartesian coordinates and is projected gnomonically or conformally on the sphere.
} 
Yin-Yang - or icosahedral grids ${ }^{3}$. A review of the different grids can be found in Staniforth and Thuburn (2012) and Williamson (2007). However, those approaches lose the latitudinal regularity arising from the rotation of the Earth. Furthermore, they require specific treatments at the singularities of the adopted polygons, which may also induce resolution clustering near these points. On the plus side, they allow for the implementation of more accurate algorithms than the ones on reduced latitude-longitude grids. This last point is especially important for weather and climate models that solve the nonlinear momentum equation, but is less stringent for the two-dimensional (2-D) linear transport of trace species on the sphere.

To construct the reduced grid, one difficulty is to define a structure which avoids treating the poles as special cases, as that can impact the precision and properties of the advection algorithm. Thus we have chosen to adopt a semi-structured approach. The grid is not regular as the number of cells varies with the latitude, but the coordinates of cell interfaces can be computed algebraically. We thus avoid storing a list of neighbors as an irregular unstructured grid would normally require, hence decreasing memory costs. This was done to anticipate future parallel architectures, which may have less memory capacity per core than current systems.

Our goal is to use an adequate algorithm exploiting the grid features to achieve efficiency and scalability on massively parallel architectures. Fine control over parallelization was obtained using the Message Passing Interface (MPI) library. In that context, the advection scheme must be chosen as to balance its accuracy vs. the volume of the required parallel communications. Furthermore, grid properties were carefully studied to improve the parallel version. In particular, a custom domain decomposition consistent with our grid was designed.

The present paper is organized as follows. Section 2 lists the basic equations and numerical methods used to solve the advection of the chemical species. In Sect. 3, results from standard test cases for advection of tracer on the sphere are reported. Those cases were chosen from the test case suite proposed by Lauritzen et al. (2012). Section 4 gives details on the model implementation on parallel architectures and the results of parallel scalability experiments. In Sect. 5, we summarize the results obtained and discuss the possible extension of our method.

\section{Numerical scheme}

\subsection{Finite-volume formulation}

Our model is based on a finite-volume method to integrate the tracer advection equation. This is performed on a bidimensional discrete grid on the sphere, which is described

\footnotetext{
${ }^{3} \mathrm{An}$ icosahedra is subdivided until the desired resolution and projected on the sphere.
}

in more detail in Sect. 2.2. In each grid cell, the tracer concentration changes according to the divergence of the fluxes at the cell boundaries. This comes from the flux form of the continuity and tracer conservation equations:

$$
\begin{aligned}
& \frac{\partial \rho}{\partial t}+\nabla \cdot(\rho \boldsymbol{V})=0, \\
& \frac{\partial \rho q}{\partial t}+\nabla \cdot(\rho q \boldsymbol{V})=0,
\end{aligned}
$$

where $\rho$ is the air density, $q$ the tracer mixing ratio and $V$ the winds vector field. Equations (1) and (2) are first integrated over a cell area $\mathcal{A}$. With $\partial \mathcal{A}$ noted as the cell boundary and $\boldsymbol{n}$ as the local normal pointing outward, the divergence theorem yields

$$
\begin{aligned}
& \frac{\partial m}{\partial t}=\frac{\partial}{\partial t} \int_{\mathcal{A}} \rho=\int_{\partial \mathcal{A}}(\rho \boldsymbol{V} \cdot \boldsymbol{n} \mathrm{d} S), \\
& \frac{\partial m_{r}}{\partial t}=\frac{\partial}{\partial t} \int_{\mathcal{A}} \rho q=\int_{\partial \mathcal{A}}(\rho q \boldsymbol{V} \cdot \boldsymbol{n} \mathrm{d} S),
\end{aligned}
$$

where $m$ is the total air mass in a cell and $m_{r}$ is the total tracer mass in a cell. The right-hand side of Eqs. (3) and (4) can be seen as the integral of all the fluxes across the cell boundaries. This formulation gives a conservative scheme when the same fluxes are used for upstream and downstream adjacent cells.

The above equations are then integrated in time during a time step:

$$
\begin{aligned}
& m^{n+1}=m^{n}-\int_{t_{n}}^{t_{n+1}}\left(\int_{\partial \mathcal{A}} \rho \boldsymbol{V} \cdot \boldsymbol{n} \mathrm{d} S\right) \mathrm{d} t, \\
& m_{r}^{n+1}=m_{r}^{n}-\int_{t_{n}}^{t_{n+1}}\left(\int_{\partial \mathcal{A}} \rho q \boldsymbol{V} \cdot \boldsymbol{n} \mathrm{d} S\right) \mathrm{d} t .
\end{aligned}
$$

Equation (5) can be omitted for non-divergent flows since the divergence of the mass fluxes is null and the mass inside the cells is constant: $[m]^{n+1}=[m]^{n}$. In this paper, we only consider non-divergent flows. As such, winds are corrected to be divergence-free in a preprocessing step, as explained in Sect. 2.3. Handling divergent flows would require minor adjustments to the scheme (removing the correction of winds and adding Eq. 5 to the scheme), but this configuration was not considered as a typical use case of CTMs, where largescale 3-D winds can be considered divergence-free.

There are many options to evaluate the tracer mass fluxes at the cell boundary. These fluxes are approximated as the air mass fluxes multiplied by the mean tracer ratio $\widehat{q}$ crossing the interface. The simplest approach to evaluate $\widehat{q}$ was introduced by Godunov (Godunov et al., 1961), who considered $\widehat{q}$ as constant within each upstream cell. The resulting scheme is conservative and monotonicity preserving but very diffusive. Improvements to the Godunov scheme were introduced 

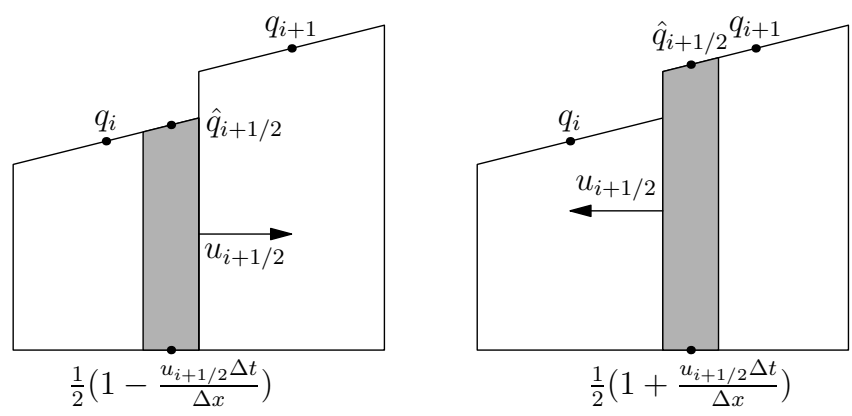

Figure 1. Van Leer scheme for positive (left) and negative (right) winds. The distribution of the tracer is shown as a linear distribution (broken line). The grey area is the quantity of tracer passing through the interface during a time step.

by van Leer in van Leer (1977), where $q$ is now approximated by a non-constant polynomial function. Depending on the polynomial degree used, i.e., the order moments of the distribution of $q$ inside the cell, van Leer obtained several schemes (up to six) that varied in complexity. A review of the different possible options can be found in Rood (1987) and Hourdin and Armengaud (1999). In general, accuracy is found to increase when higher-order moments are used, but the price to pay lies in larger computational and memory costs. Using higher moments requires a larger number of grid points to compute the derivatives, which increases communication volumes when domain decomposition techniques are used for parallel clusters (see Sect. 4).

For our model, we have adopted a first-order reconstruction, the van Leer scheme (noted as van Leer I in the original paper). The distribution of $q$ in the cells is approximated by a linear function in latitudinal and meridional directions. The slope of the linear function is computed as a finite difference using the values of $q$ within the nearest cells. In that configuration, the scheme is second-order accurate in space. To extend the algorithm to multiple dimensions, a time-splitting scheme is used. Equations (5) and (6) are first integrated in the zonal direction:

$\widetilde{m}_{i}^{n+1}=m_{i}^{n}+U_{i-1 / 2}-U_{i+1 / 2}$,

$\left(\widetilde{m_{r}}\right)_{i}^{n+1}=\left(m_{r}\right)_{i}^{n}+F_{i-1 / 2}-F_{i+1 / 2}$,

where $U$ and $F$ are the air and tracer fluxes, respectively, across the borders orthogonal to the chosen direction during a time step. With these notation, tracer fluxes are approximated as $F_{i+1 / 2} \approx \widehat{q}_{i+1 / 2} u_{i+1 / 2}$. Figure 1 illustrates the reconstruction of $\widehat{q}_{i+1 / 2}$. The linear distribution represents the tracer distribution in the 1-D case for cells $i$ and $i+1$. Finding $\widehat{q}_{i+1 / 2}$ depends on the wind direction: for outward winds $\left(u_{i+1 / 2}>0\right)$, the grey area in the left diagram will move from cell $i$ to cell $i+1$. Then the mean tracer ratio corresponding to this flux is computed from the distribution in cell $i$. The same can be applied for inward fluxes, resulting in $\widehat{q}_{i+1 / 2}= \begin{cases}q_{i}+\left(1-\frac{u_{i+1 / 2} \Delta t}{\Delta x}\right)(\delta q)_{i} & \text { if } \quad u_{i+1 / 2}>0, \\ q_{i+1}-\left(1+\frac{u_{i+1 / 2} \Delta t}{\Delta x}\right)(\delta q)_{i+1} & \text { otherwise, }\end{cases}$

where $\delta q$ is the slope of the linear reconstruction and $u_{i+1 / 2}$ the wind at the interface. $\Delta t$ and $\Delta x$ are the time step and cell spacing, respectively.

This first advection step gives us the intermediate mass value $\widetilde{m}$ and tracer value $q^{\prime}=\widetilde{m} / \widetilde{m_{r}}$. These new values are then used to integrate Eq. (6) in the meridional direction. As the grid is unstructured, mass and tracer fluxes in the northsouth direction have to be evaluated for all the neighbors of each cell. This is detailed in Sect. 2.3.

It should be noted that other time-splitting schemes are available. Another approach is to use a zonal-meridional advection during a time step and meridional-zonal for the next. It is also possible to compute both zonal-meridional and meridional-zonal advection and then use their mean as the final value. For the complete description of each method, see Machenhauer et al. (2009). Either way, the final bidimensional algorithm is second-order accurate. In Pangolin, all three time-splitting schemes have been tested using the numerical order of convergence test (see Sect. 3.3). It was found the choice of the time-splitting algorithm has little impact on accuracy.

To ensure monotonicity of the solution and to prevent numerical oscillations, van Leer introduced a slope limiter. The idea is to limit the slope value within a given cell such as the tracer value in that cell does not exceed the mean value of the adjacent cells. This is more restrictive than limiting the fluxes to ensure that the tracer values remain between the maximum-minimum values of the adjacent cells but is easier to implement. The slope is given by

$$
\begin{aligned}
& (\delta q)_{i}=\min \left(\frac{1}{2}\left|q_{i+1}-q_{i-1}\right|, 2\left|q_{i+1}-q_{i}\right|, 2\left|q_{i}-q_{i-1}\right|\right) \\
& \quad \times \operatorname{sign}\left(q_{i+1}-q_{i}\right)
\end{aligned}
$$

if $q_{i}$ lies in between $q_{i-1}$ and $q_{i+1}$, and $(\delta q)_{i}=0$ otherwise. As discussed by Hourdin and Armengaud (1999), the slope limiter efficiently damps the numerical oscillations but introduces more diffusion of the numerical solutions. For the test cases reported in this study, the slope limiter appears to have little impact on the accuracy of the numerical solutions.

\subsection{Grid}

The grid used in Pangolin is completely defined by the number of cells at the North Pole and the number of latitudes $n_{\text {lat }}$ on a hemisphere. The Southern Hemisphere is simply constructed in the same way as the Northern Hemisphere. To find the number of cells at the North pole, we can write the equality between cell areas at the pole and at the Equator. If we consider squared cells at the Equator, like the ones on 



Figure 2. Grid used in Pangolin with 20 latitudes: orthographic projection (left) and Robinson projection, with the six identical zones highlighted (right).

a regular latitude-longitude grid, and small latitudinal spacing, the number of cells is approximately $\pi$. Thus we set the number of cells at the poles to 3 .

At a given latitude, all cells have the same area. We can then compute the number of cells for all latitudes. For maximum flexibility, latitudinal and longitudinal spacings are no longer assumed identical. Let us consider the area of cell $(i, j)$, noted $\mathcal{A}_{i}$ as it does not depends on $j$. Let us note $\phi_{i}$ as the colatitude and $\lambda_{i j}$ as the position of the south and east cell borders. As we assume that the cell spacings are constant, we can write $\phi_{i}=i \Delta \phi_{i}$ and $\lambda_{i j}=j \Delta \lambda_{i}$. The area is defined on $\Omega_{i j}=\left[\lambda_{j}, \lambda_{j+1}\right] \times\left[\phi_{i-1}, \phi_{i}\right]$, so in spherical coordinates we have

$$
\begin{aligned}
\mathcal{A}_{i} & =\iint_{\Omega_{i j}} r^{2} \sin \phi \mathrm{d} \lambda \mathrm{d} \phi \\
& =r^{2} \Delta \lambda_{i}\left(\cos \left(\phi_{i-1}\right)-\cos \left(\phi_{i-1}+\Delta \phi_{i}\right)\right) .
\end{aligned}
$$

Areas are preserved so $\mathcal{A}_{i}=\mathcal{A}_{1}$ :

$\Delta \lambda_{i}=\Delta \lambda_{1} \frac{1-\cos \left(\Delta \phi_{1}\right)}{2 \sin \left(\frac{\Delta \phi_{i}}{2}\right) \sin \left(\left(i-\frac{1}{2}\right) \Delta \phi_{i}\right)}$.

Noting $n_{i}$ the number of cells at colatitude $i$, we get

$\frac{n_{i}}{n_{1}}=\left\lfloor\frac{\Delta \lambda_{1}}{\Delta \lambda_{i}}\right\rfloor=\left\lfloor\frac{2 \sin \left(\frac{\Delta \phi_{i}}{2}\right) \sin \left(\left(i-\frac{1}{2}\right) \Delta \phi_{i}\right)}{1-\cos \left(\Delta \phi_{1}\right)}\right\rfloor$.

Now let us assume $\forall i, \Delta \phi_{i}$ and $\left(i-\frac{1}{2}\right) \Delta \phi_{i}$ are small enough:

$\frac{n_{i}}{n_{1}} \approx\left\lfloor\frac{2 \frac{\Delta \phi_{i}}{2}\left(i-\frac{1}{2}\right) \Delta \phi_{i}}{\frac{\Delta \phi_{1}^{2}}{2}}\right\rfloor=2 i-1$.
Finally, we can define the number of cells for the whole grid, with $2 n_{\text {lat }}$ latitudes, as

$n_{i}= \begin{cases}3(2 i-1) & \text { if } 1 \leq i \leq n_{\text {lat }}, \\ n_{2 n_{\text {lat }}-i+1} & \text { otherwise. }\end{cases}$

It follows that the total number of cells on the grid is $6 n_{\text {lat }}^{2}$. As an illustration, the grid is shown in Fig. 2.

The previous formula is a sound approximation for area preservation near the poles and when latitudinal spacing is constant. In practice, we consider the approximation as reasonable up to $75^{\circ}$ : the relative error is then less than $1 \%$. At lower latitudes, the error increases, with a maximum of $56 \%$ at the Equator. So the grid used in Pangolin gives higher resolutions at the Equator than at the poles. One way around this issue is to truncate the number of cells at a given threshold. As a comparison, Fig. 3 shows the number of cells for Pangolin with the "exact" and truncated version. By "exact", we mean the number of cells comes from the area-preservation formulae without any approximations for $\Delta \phi_{i}$. Furthermore, to truly preserve the cell areas, we should use a variable latitudinal spacing. However, the distortion due to a constant latitudinal spacing was found to be acceptable and much less pronounced compared with a regular latitude-longitude grid.

The formula given in Eq. (10) allows us to easily determine the coordinates of the cell neighbors in each zone. The grid used in Pangolin has four axes of symmetry $-\lambda=0$, $\lambda=120, \lambda=240^{\circ}$ and the Equator - and so the grid can be split into six identical zones, numbered with regard to west to east and north to south as shown in Fig. 2. The following formulae to compute the position of the cell neighbors are given for the first zone - i.e., $\left[90^{\circ}, 0\right] \times\left[0,120^{\circ}\right]$. The formulae on zones 2 and 3 are obtained by adding the proper offset. In the Southern Hemisphere (zone 4 to 6), the north and south formulae are simply inverted. The zonal neighbors for cell $(i, j)$ are simply $(i, j-1)$ and $(i, j+1)$. For the first zone, its north and south neighbors are respectively 


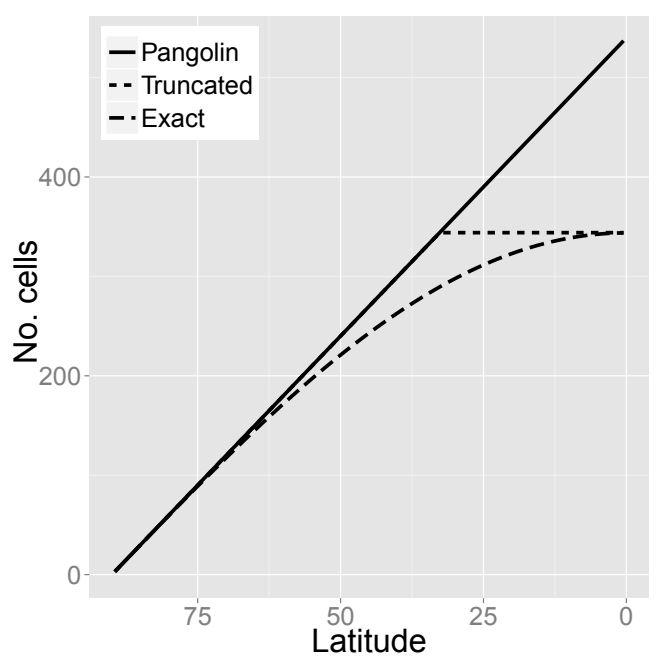

Figure 3. Number of cells for the grid used by Pangolin on one hemisphere with 90 latitudes (solid line). The truncated and "exact" version are shown as dotted and dashed lines, respectively.

$\left\{\left(i-1, j_{1}\right), \ldots,\left(i-1, j_{2}\right)\right\}$ and $\left\{\left(i+1, j_{3}\right), \ldots,\left(i+1, j_{4}\right)\right\}$, with

$j_{1}=\left\lfloor\frac{n_{i-1}}{n_{i}}(j-1)+1\right\rfloor, \quad j_{2}=\left\lceil\frac{n_{i-1}}{n_{i}} j\right\rceil$,

$j_{3}=\left\lfloor\frac{n_{i+1}}{n_{i}}(j-1)+1\right\rfloor, \quad j_{4}=\left\lceil\frac{n_{i+1}}{n_{i}} j\right\rceil$.

From that formulation, it follows that the number of meridional neighbors is not constant, even though most of cells have two north and two south adjacent cells. Special cases include the middle of each sector (one north and three south neighbors) and its extremities (one north and two south). These figures apply for the Northern Hemisphere and must be inverted in the Southern Hemisphere.

As a consequence, computing the position of the neighbors is quite efficient, involving mostly integer operations and roundings. These computations are thus performed on the fly to reduce the storage requirements. In a more general way, the algebraic properties of the grid are exploited as much as possible. Our parallelization strategy relies heavily on the properties of the grid, as shown in Sect. 4.

\subsection{Adapting the scheme to the grid}

For winds and tracer discretization, we adapt the Arakawa C grid (Arakawa and Lamb, 1977) to our scheme, as shown in Fig. 4. To avoid interpolating the winds components during advection, winds are taken at the middle of the interfaces. Tracer concentrations are defined at the centers of the cells.

Due to the structure of the grid (Fig. 2), air and tracer fluxes need to be computed for all the neighbors of the cells as illustrated in Fig. 5. For each flux, the frontier between the current cell and its neighbor is computed algebraically using the cell neighbors formulae (Eq. 11). While there is no

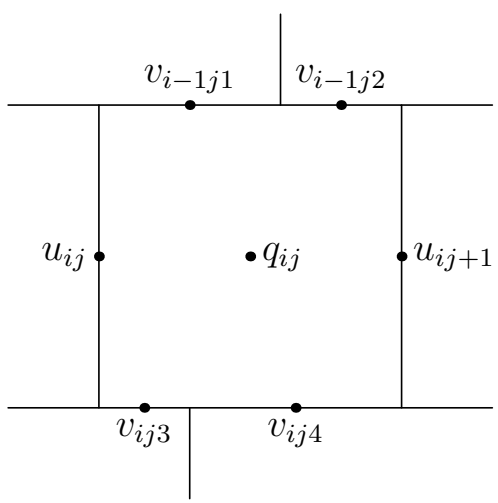

Figure 4. Discretization for zonal and meridional winds ( $u$ and $v$, respectively) and tracer mixing ratio $q$.

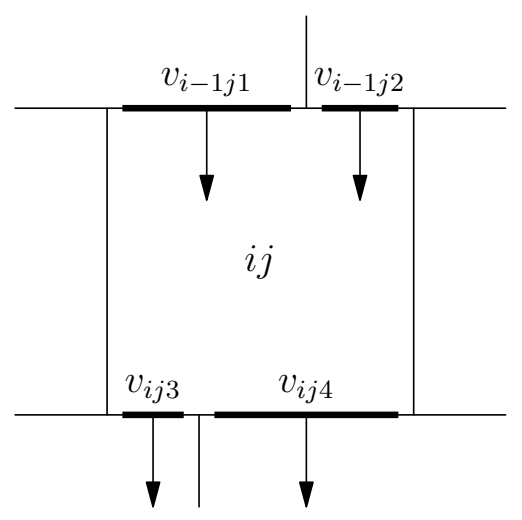

Figure 5. Meridional interfaces (bold lines) and fluxes (arrows) for cell $(i, j)$.

special treatment for zonal advection, meridional advection requires an interpolation to compute the meridional gradient. A linear interpolation in the zonal direction is computed to evaluate the value of the mixing ratio on the meridian passing through the center of the cell (see Fig. 6). These values are used to compute the meridional gradient by finite difference.

It is critical for mass preservation that winds have a null divergence, so we correct interpolated winds (both zonal and meridional) to achieve that. The first step in our correction deals with meridional winds. If we consider all the cells on a latitude circle, the total mass variation in this "band" only comes from the north and south meridional fluxes. Now, if we consider the latitude circle containing all south meridional fluxes, it constitutes a closed contour. Therefore, the wind divergence is null and the sum of all meridional winds must be zero. Meridional winds are thus corrected by removing the mean value from the interpolated values. For a future 3-D case, a preprocessing step involving vertical winds will be needed to ensure non-divergent circulation. Depending on the system of vertical coordinates used, the "mass-winds in- 
consistency" issue (see, for example, Jöckel et al., 2001) will have to be addressed.

Then we correct zonal winds to ensure that the sum of all fluxes is locally null in each cell. As meridional winds are already corrected, only east and west zonal winds of each cell must be modified. We take zonal winds at longitude 0 as a reference and browse each cell sequentially from west to east to progressively correct each of the zonal winds and ensure mass preservation.

Finally, we need to take care that fluxes in a given direction do not completely empty the cells during an advection step. For each cell, a local advective Courant number restricts the time step in order to avoid this situation. As advection is performed sequentially in two different directions, we define two unidimensional local Courant numbers. Then the global Courant number $C$ is simply defined as the most restrictive condition on all cells:

$C=\max _{i j}\left(\frac{u_{i j} \Delta t}{\Delta \phi_{i j}}, \frac{\Delta t \sum_{k \in V_{i j}} v_{k} \Delta \lambda_{k}}{\Delta \phi_{i j} \sum_{k \in V_{i j}} \Delta \lambda_{k}}\right)$,

where $V_{i j}$ is the set of meridional neighbors for the cell $(i, j)$ and $\Delta \lambda_{k}$ is the interface size between the cell and its neighbor. For the tests in this paper, we use $C_{\max }=0.96$ as a Courant-Friedrichs-Lewy (CFL) condition.

\section{Testing suite}

A standard 2-D testing suite to check the accuracy and properties of a transport model was proposed in Lauritzen et al. (2012). A comparison with state-of-the-art schemes was subsequently published in Lauritzen et al. (2013), which offers a convenient benchmark to compare transport models on the sphere. From it, we have extracted a subset of the models and cases which we felt were relevant to Pangolin. In Lauritzen et al. (2013), the different grids were compared with a constant resolution at the Equator. In the present paper, we retain simulations performed with a constant total number of cells. The number of cells in each model was computed using the resolution at the Equator given in the appendix of Lauritzen et al. (2013). As a summary, Table 1 contains the formulae used and gives an idea of the size of each grid in comparison with Pangolin.

\subsection{Models features}

The models were chosen as their spatial order is similar to Pangolin. They are implemented on both regular and nonregular grids and provide a basis for a comparison between semi-Lagrangian, finite-volume and wave propagation methods. A summary is given in Table 2. Other features are described below:

- FARSIGHT is a grid-point semi-Lagrangian model, running on parallel architectures.
Table 1. For a given resolution at the Equator, we compare the total number of cells of each model $n_{\text {model }}$ vs. the total number of cells of Pangolin $n_{\text {pangolin. }}$.

\begin{tabular}{lcc}
\hline Model & $n_{\text {model }} / n_{\text {pangolin }}$ & $n_{\text {model }}$ \\
\hline FARSIGHT & 2 & $6 \cdot(90 / \Delta \lambda)^{2}$ \\
CLAW & 0.68 & $2 \cdot(90 / \Delta \lambda)^{2}$ \\
SLFV-ML & 2.17 & NA \\
CAM-FV & 2.7 & $\lfloor 360 / \Delta \lambda\rfloor \cdot\lfloor 180 / \Delta \lambda\rfloor$ \\
UCISOM & 2.7 & $\lfloor 360 / \Delta \lambda\rfloor \cdot\lfloor 180 / \Delta \lambda\rfloor$ \\
Pangolin & 1 & $6 \cdot\lceil 0.5 \cdot\lceil 120 / \Delta \lambda\rceil+1\rceil^{2}$ \\
\hline
\end{tabular}

- CLAW uses a wave propagation technique with a firstorder method (donor cell upwind) in each direction.

- SLFV-ML (Slope Limited Finite Volume scheme with Method of Lines), a flux-form finite volume with simplified swept area and linear reconstruction.

- CAM-FV (Community Atmosphere Model FiniteVolume) is a finite-volume model on a regular latitudelongitude grid. It uses the piecewise parabolic method (PPM), with the addition of a slope and curvature limiters.

- USICOM (UC Irvine Second-Order Moments scheme) is also a flux-form finite volume. It uses an improved version of Prather's second-order moments scheme on an Eulerian regular latitude-longitude grid.

All of these models are mass preserving, so the comparison with Pangolin is relevant. CAM-FV and UCISOM are of particular interest as they also use a directional splitting algorithm. Furthermore, all models have a shape-preserving algorithm but only FARSIGHT and Pangolin do not expand the initial tracer concentration range.

\subsection{Test cases}

In this paper, we only consider the case of non-divergent and time-dependent winds. Zonal and meridional winds ( $u$ and $v$, respectively) are given by

$$
\begin{aligned}
& u(\lambda, \theta, t)=\frac{10 R}{T} \sin ^{2}\left(\lambda^{\prime}\right) \sin (2 \theta) \cos \left(\frac{\pi t}{T}\right)+\frac{2 \pi R}{T} \cos (\theta), \\
& v(\lambda, \theta, t)=\frac{10 R}{T} \sin \left(2 \lambda^{\prime}\right) \cos (\theta) \cos \left(\frac{\pi t}{T}\right)
\end{aligned}
$$

where $\theta$ is now the latitude and $\lambda$ the longitude, both in radians. $R$ is the Earth radius, $T$ is the period, set at 12 days here, and $\lambda^{\prime}=\lambda-2 \pi t / T$. With these winds, the tracer concentration first moves eastwards and is then deformed into filaments up to $t=T / 2$. After that, the flux is inverted and the tracer continues to move to the east until it comes back to its initial distribution at $t=T$. 
These winds provide an easy way to compute the errors as the solution after a full period can be simply compared with the initial concentration. We will use the same normalized errors as in Lauritzen et al. (2012):

$\ell_{2}(q)=\sqrt{\frac{\mathcal{I}\left(\left(q-q_{0}\right)^{2}\right)}{\mathcal{I}\left(q_{0}^{2}\right)}} \quad$ and $\quad \ell_{\infty}(q)=\frac{\max _{\forall \lambda, \theta}\left|q-q_{0}\right|}{\max _{\forall \lambda, \theta}\left|q_{0}\right|}$,

where $q=q(\lambda, \theta, t)$ is the tracer concentration and $q_{0}$ the initial concentration. Also, $\mathcal{I}$ is defined as the global integral:

$\mathcal{I}(q)=\frac{1}{4 \pi} \int_{0}^{2 \pi} \int_{-\pi / 2}^{\pi / 2} q(\lambda, \theta, t) \cos \theta \mathrm{d} \lambda \mathrm{d} \theta$.

For our model, the tracer mixing ratio is approximated as linear functions in a cell. Thus the mean value corresponds to the value at the middle of the cell, so the integral is approximated by $\mathcal{I}(q)=\sum_{i, j} \widehat{q}_{i j} \mathcal{A}_{i j}$, where $\widehat{q}_{i j}$ is the tracer mean value in cell $(i, j)$ and $\mathcal{A}_{i j}$ its area given by Eq. (9).

Two initial conditions are used here: a sum of two Gaussian hills and a sum of two cosine bells. We note $\left(\lambda_{1}, \theta_{1}\right)=$ $(5 \pi / 6,0)$ and $\left(\lambda_{2}, \theta_{2}\right)=(7 \pi / 6,0)$ as the coordinates of the two "centers" used below. Gaussian hills are defined as

$q(\lambda, \theta)=h_{1}(\lambda, \theta)+h_{2}(\lambda, \theta)$.

Noting $h_{\max }=0.95$ and $b=5$, for $i=1,2$ we have

$h_{i}(\lambda, \theta)=h_{\max } e^{-b\left(\left(X-X_{i}\right)^{2}+\left(Y-Y_{i}\right)^{2}+\left(Z-Z_{i}\right)^{2}\right)}$,

where $X, Y, Z$ are the Cartesian coordinates of $(\lambda, \theta)$ and $X_{i}, Y_{i}, Z_{i}$ are the Cartesian coordinates of $\left(\lambda_{i}, \theta_{i}\right)$ for $i=$ 1,2 . Cosine bells are defined by

$q(\lambda, \theta)=\left\{\begin{array}{lll}b+c \times h_{1}(\lambda, \theta) & \text { if } & r_{1}<r, \\ b+c \times h_{2}(\lambda, \theta) & \text { if } & r_{2}<r, \\ b \text { otherwise, } & & \end{array}\right.$

with the background value $b=0.1$ and amplitude $c=0.9$. Noting $h_{\max }=1$ and $r=R / 2$, we also have

$\forall i=1,2, \quad h_{i}(\lambda, \theta)=\frac{h_{\max }}{2}\left(1+\cos \left(\pi \frac{r_{i}}{r}\right)\right)$,

where the $r_{i}$ are the great-circle distances to $\left(\lambda_{i}, \theta_{i}\right)$ on the sphere:

$r_{i}(\lambda, \theta)=R \arccos \left(\sin \theta_{i} \sin \theta+\cos \theta_{i} \cos \left(\lambda-\lambda_{i}\right)\right)$.

Pangolin results for the Gaussian hills and cosine bells test cases are shown in Fig. 7 at $t=0$, half the period and after a full period. The shape of the tracer distribution is well preserved but numerical diffusion contributes to a decrease in the tracer maxima, as it appears at $t=T / 2$ and $t=T$. To compute the numerical order of convergence in Sect. 3.3, results at $t=0$ and $t=T$ will be used, while the preservation of filaments in Sect. 3.4 is computed using the results at $t=0$ and $t=T / 2$.

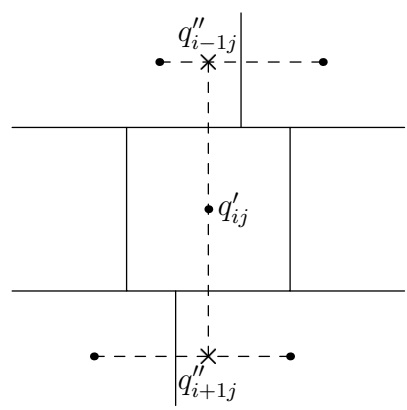

Figure 6. Zonal interpolation to compute the meridional gradient of $q_{i j}^{\prime}$

\subsection{Numerical order of convergence}

The aim of this test is to check the rate at which numerical error decreases when resolution increases. Ideally, this rate should be close to the theoretical order of convergence. The Gaussian hills test case is used here, as it provides an infinitely smooth function. Results are plotted in Fig. 8, where $\ell_{2}$ and $\ell_{\infty}$ are plotted with a varying number of cells. When available, the impact of shape-preserving filters is also represented on the plot, with the exception of UCISOM. For this choice of models, it does not reduce errors in a significant way as can be expected from lower-order models.

For errors at low and medium resolutions, Pangolin is quite close to the other models, with the exception of UCISOM. However, the errors with a large number of cells are lower for models other than Pangolin. One possible explanation is the loss of accuracy due to the interpolation when computing the meridional gradient. In general, the order of convergence of Pangolin is lower. To quantify that, we use numerical optimal order of convergences corresponding to the errors $\ell_{2}$ and $\ell_{\infty}$. They result from a least-squares linear regression on the errors plotted vs. the resolution at the Equator:

$\log \left(\ell_{i}\right)=-\kappa_{i} \log (\Delta \lambda)+b_{i} \quad$ with $\quad i=2, \infty$

For Pangolin, the regression is applied after the optimal convergence has been reached. This corresponds to longitudinal resolutions at the Equator in the range $\left[0.75^{\circ}, 0.1875^{\circ}\right]$. The final numerical convergence rates are shown in Fig. 9.

This selection of models and parameters shows that theoretical order is not achieved for all models. For most of them, using a different Courant number does not improve the convergence speedup, with the exception of FARSIGHT and CAM-FV. Using a Courant number of 10.4 (1.2) greatly improves the result of FARSIGHT (CAM-FV) with a Courant number of $1.4(0.2)$.

Furthermore, we can see the numerical order of convergence of Pangolin is lower than other models. This is not surprising when comparing with similar finite-volume schemes such as CAM-FV and UCISOM, which use higherorder schemes in one or more directions. We studied this 
Table 2. Summary of the models used as a comparison: name, implementation grid, the total number of cells vs. Pangolin, and the time scheme.

\begin{tabular}{llcll}
\hline Model & Grid & $\begin{array}{c}\text { Formal } \\
\text { accuracy }\end{array}$ & $\begin{array}{l}\text { Time } \\
\text { scheme }\end{array}$ & Reference \\
\hline FARSIGHT & Gnomonic cubed sphere & 2 & Third order & White and Dongarra (2011) \\
CLAW & Two-patch sphere grid & 2 & Euler forward & LeVeque (2002) \\
SLFV-ML & Icosahedral-hexagonal & 2 & Runge-Kutta 3rd-order TVD & Miura (2007) \\
CAM-FV & Regular lat-long & 2 & Euler forward & Collins and Rasch (2004) \\
UCISOM & Regular lat-long & 2 & Euler forward & Prather (1986) \\
Pangolin & Pangolin & 2 & Euler forward & This paper \\
\hline
\end{tabular}
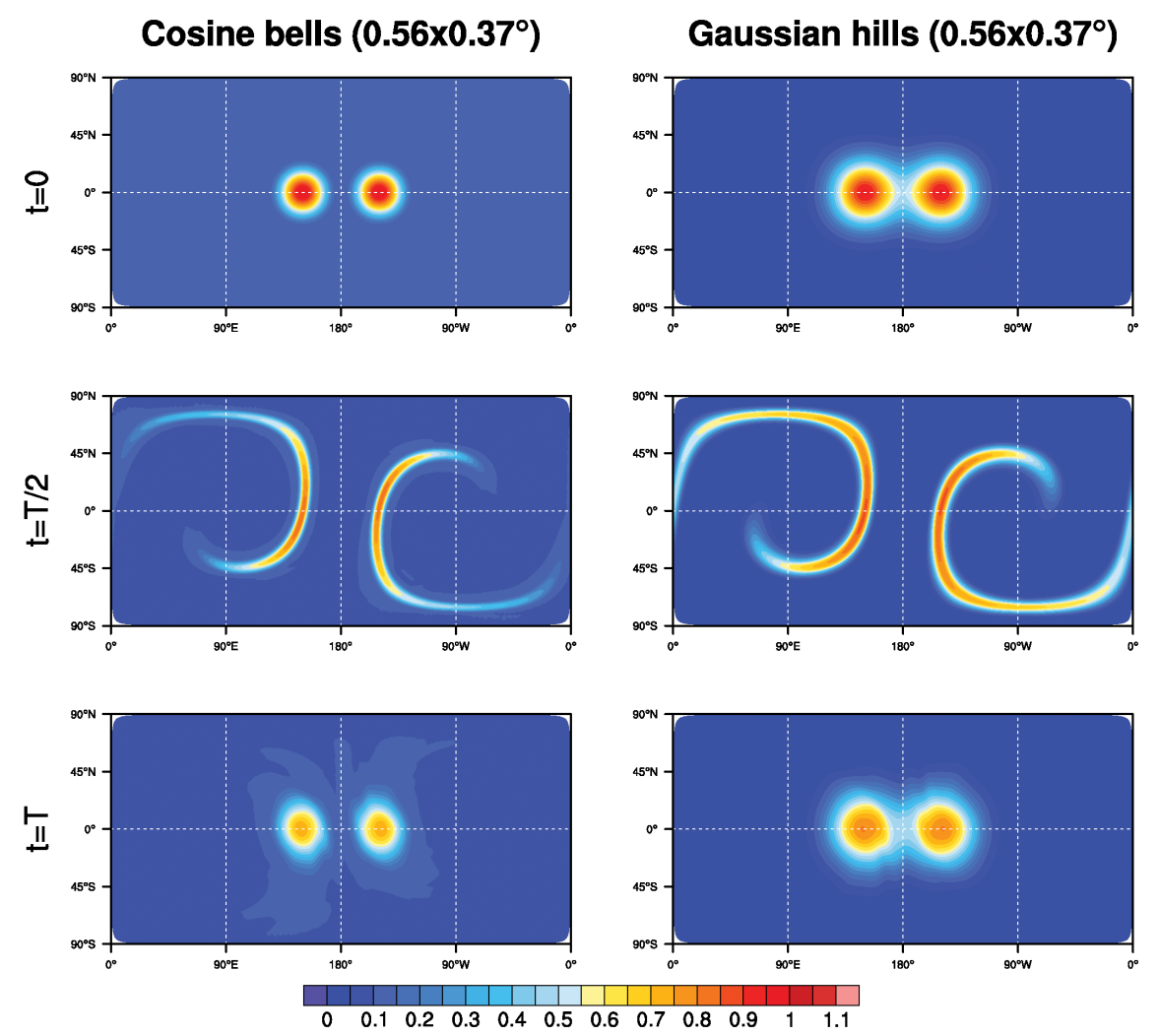

Figure 7. Cosine bells and Gaussian hills results for $t=0, t=T / 2, t=T$ (top to bottom) with Pangolin. The initial distribution is first deformed into filaments and then advected back to its initial position. The Equator resolution is $0.56^{\circ} \times 0.37^{\circ}$. For these plots, Pangolin data are interpolated to a regular latitude-longitude grid.

issue further using solid-body rotation test cases (described in Williamson et al., 1992) and found that the accuracy was limited by the linear interpolation done for the meridional gradient. When the axis of the solid-body rotation matches the polar axis, accuracy is close to second order, whereas the level of accuracy decreases when the axis is in the equatorial plane. In practice, Pangolin needs finer resolutions, as shown in the following test cases, to match the accuracy of other models.

\subsection{Preservation of filaments}

Realistic distributions will most likely be deformed into filaments when the tracer material is stretched and gradients are increased. For some applications, it is important to check how well these filaments are preserved. In the cosine bells test case, the initial concentration is deformed into thin filaments up to $t=T / 2$, before being advected to the initial position. Diagnostics are thus computed at $T / 2$.

Let us consider the area where the tracer is greater than a given threshold $\tau$. For non-divergent flows and for all thresholds, if this area is not preserved at all times, it sug- 


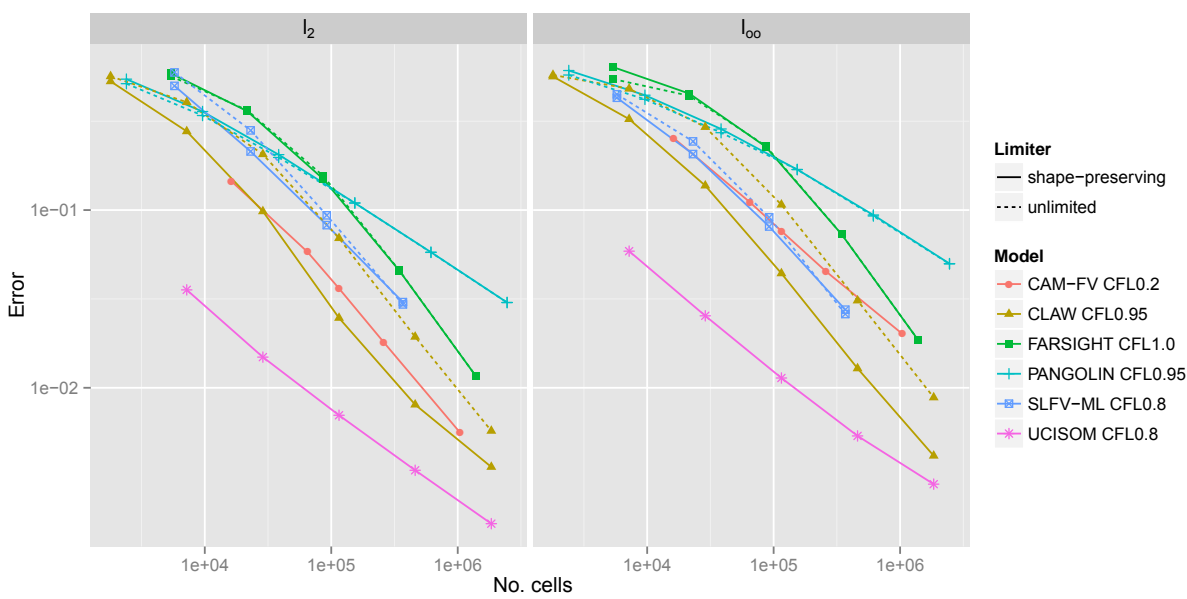

Figure 8. Numerical order of convergence for both error measures. These are computed using Gaussian hills after a full rotation. When available, the models are shown with and without the shape-preserving limiters.

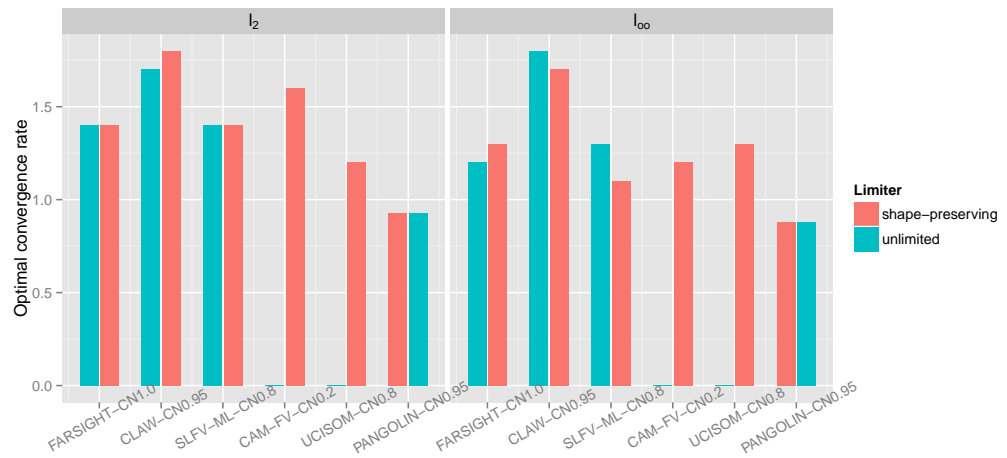

Figure 9. Optimal order of convergence computed by a least-squares regression on data from Fig. 8. Some models did not offer data without shape-preserving limiters (CAM-FV, UCISOM).

gests filaments are degraded. This leads to the definition of the following diagnostic:

$\ell_{\mathrm{f}}=\left\{\begin{array}{l}100 \times \frac{A(\tau, t)}{A(\tau, 0)} \\ 0 \quad \text { otherwise, }\end{array}\right.$

where $A(\tau, t)$ is the surface area for which the tracer ratio at time $t$ is greater or equal to $\tau$. For Pangolin and other Eulerian schemes, $A(\tau, t)$ is defined as the sum of the cell areas where $q(t) \geq \tau$. Thus the closest $\ell_{\mathrm{f}}$ is to 100 , the better the filaments are preserved.

Results are shown in Fig. 10. All models except Pangolin are compared with different numbers of cells but with the same resolution of $0.75^{\circ}$ at the Equator. Pangolin is shown with two different numbers of cells. The first case (shown as grey solid line) has a resolution of approximately $0.75^{\circ}$ at the Equator. The second one (shown as black solid line) is a higher-resolution version $\left(0.1^{\circ}\right.$ at the Equator) which approaches the results of more accurate models.

The behavior of the models on non-latitude-longitude grids (all models except UCISOM and CAM-FV) is typical of diffusive schemes. They tend to diffuse the base of the distribution and reduce the maxima, thus leading to an increase in $\ell_{\mathrm{f}}$ for small $\tau$ and a decrease in $\ell_{\mathrm{f}}$ for large $\tau$. Another piece of information we can extract from this is the alteration of gradients. Here CAM-FV can be seen to have $\ell_{\mathrm{f}}>100$ for large $\tau$, which results from gradient steepening. On the other hand, schemes on non-regular grids have a smooth and decreasing profile, showing that the scheme diffusion is also smooth and continuous.

Furthermore, the shape from the diagnostic for Pangolin is quite close to models using non-regular latitude-longitude grids (CLAW, FARSIGHT, SLFV-ML). The behavior is typical of diffusive schemes, where $\ell_{\mathrm{f}}$ is increased for lower threshold values and decreased for higher $\tau$. Pangolin is less accurate than these models, but similar accuracy can be achieved using a finer resolution. Nevertheless, the models using a regular latitude-longitude grid, namely UCISOM and CAM-FV, are the most accurate for this test. 


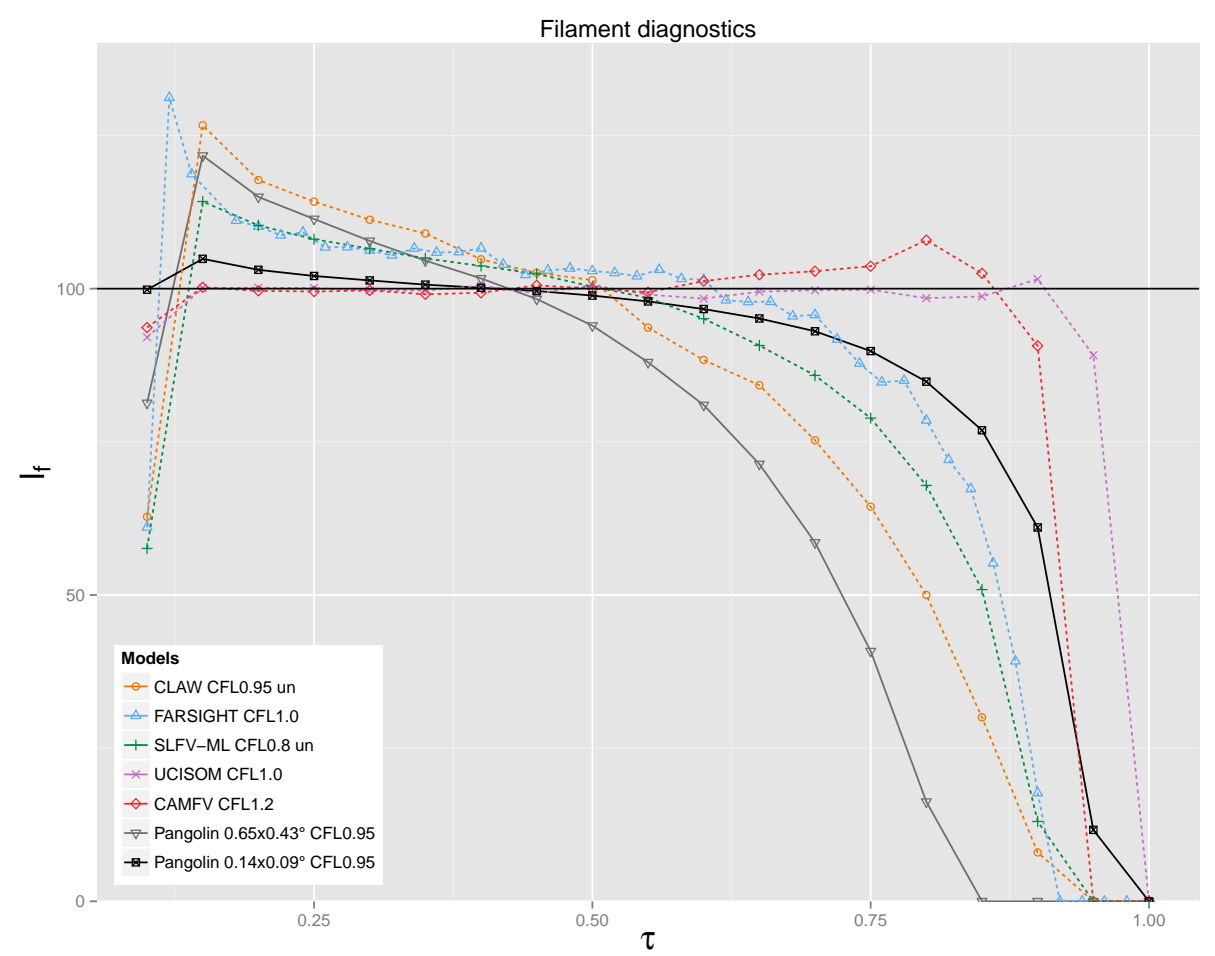

Figure 10. Filament diagnostics between for Pangolin (solid line) and other models (dashed line). By default, the shape-preserving version is used. Only CLAW and SLFV-ML use an "unlimited" version.

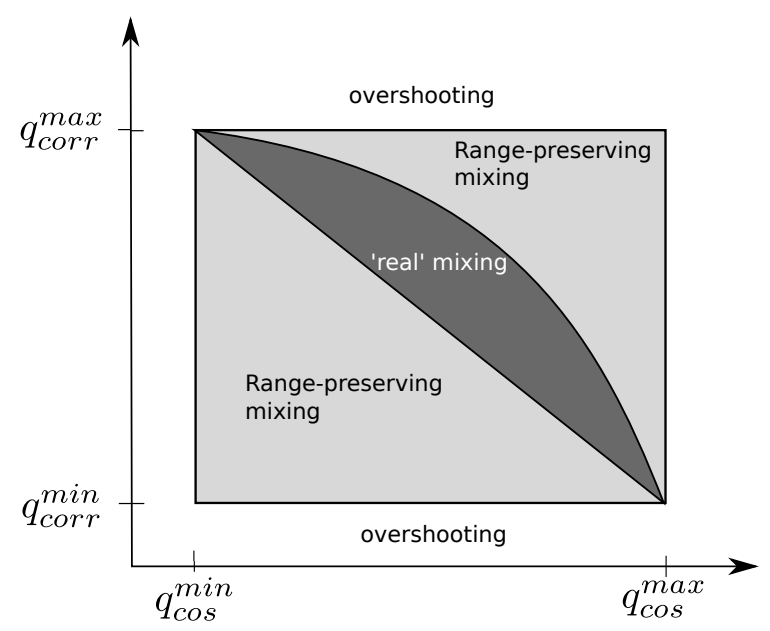

Figure 11. Different types of mixing when plotting the correlated concentration against the cosine bells distribution.

\subsection{Preservation of preexisting relations}

When advecting several correlated tracers, numerical transport schemes should preserve the relations between them. However, errors due to numerical diffusion can modify these relations and introduce a "mixing". This is by no means an unphysical feature as real-life tracers can undergo some mixing too, either by chemical reactions or diffusion. This test aims at assessing the amount of unphysical mixing. To that end, we follow Lauritzen et al. (2012) and use a first tracer with the cosine bells initial condition $q_{\mathrm{cos}}(t=0)$ and a second tracer correlated to the first:

$q_{\mathrm{corr}}(t=0)=-0.8 q_{\mathrm{cos}}^{2}(t=0)+0.9$.

After a half-period of advection using the non-divergent winds for each case, we plot $q_{\mathrm{corr}}(t=T / 2)$ against $q_{\mathrm{cos}}(t=$ $T / 2$ ) as a scatterplot. Depending on the position of the points, we can then check the mixing level. An illustration of the different zones is given in Fig. 11. The shaded convex area in the figure corresponds to "real" mixing as it contains all the lines between two points on the curve corresponding to Eq. (12). The light-grey area is not a physical mixing but is still in the initial range. Everything outside the box is overshooting, which may result in unphysical concentrations such as negative values.

Results are shown in Figs. 12 and 13. All unlimited schemes present some overshooting in the upper left corner of the figure, which is then removed with a shape-preserving filter. Also, all schemes, with the exception of UCISOM, present real mixing. For this selection of models, only FARSIGHT and CAM-FV have range-preserving unmixing. For FARSIGHT, this can be removed with a larger Courant number. In that case, its accuracy is on the same level as UCISOM. Concerning accuracy, the closer the points lie to the curved defined in Eq. (12), the more the scheme preserves the 


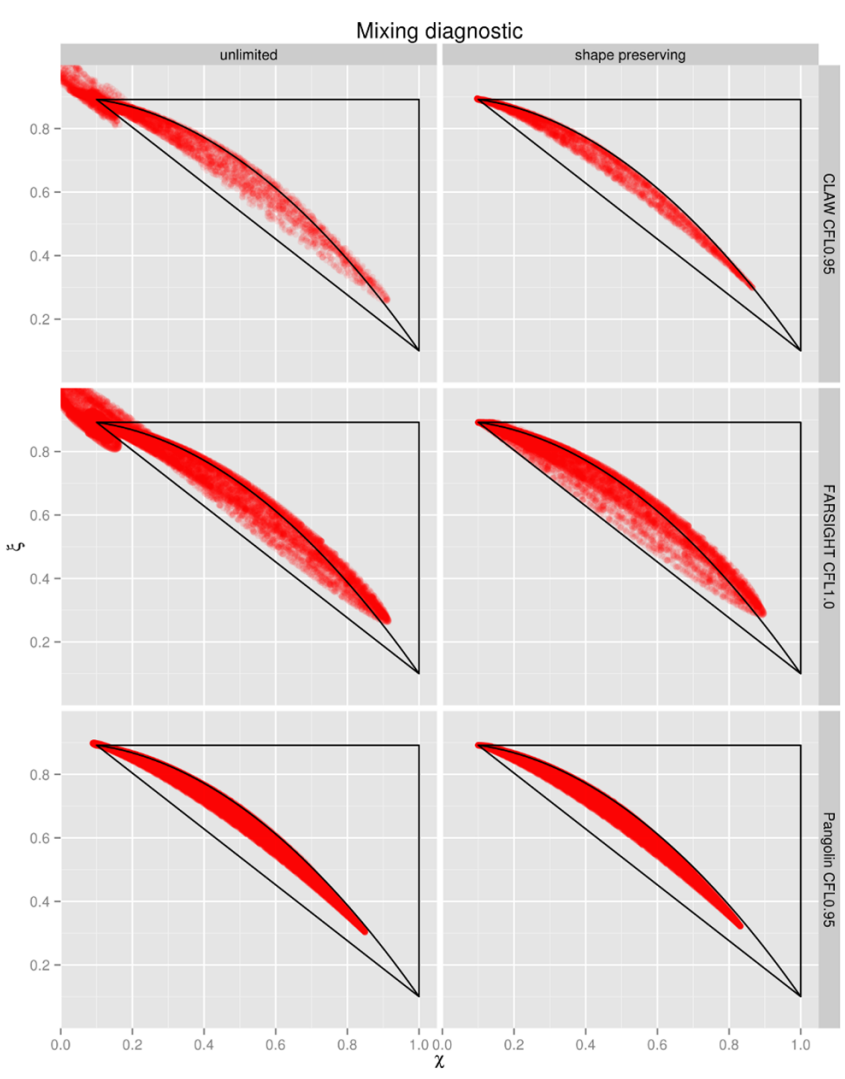

Figure 12. Mixing plots for both unlimited and shape-preserving versions. The resolution is set at $0.75^{\circ}$ at the Equator, except for Pangolin, which has $0.376^{\circ}$.

initial relation. In that respect, UCISOM is the most accurate model of this selection, followed by Pangolin and CAM-FV.

\subsection{Comparing parallel performances}

Pangolin was designed with scalability and parallel performances in mind, thus leading to the choice of a smaller stencil than the models presented here. From the results presented above, it is clear that Pangolin can match the accuracy of other models using finer resolution. Unfortunately, comparing the parallel performances in terms of running time on multi-core architectures is difficult. Some models provide technical details about the performances - see White and Dongarra (2011) for FARSIGHT, Dennis et al. (2011) for CAM-FV or Erath and Nair (2014) and Guba et al. (2014) for some other state-of-the art schemes - but there are not enough data for a thorough comparison. We thus provide as much detail as possible on the parallel performances of Pangolin alone. In particular, Sect. 4.3 highlights the smallest size of subdomains needed for a reasonable efficiency for 2D parallel advection.

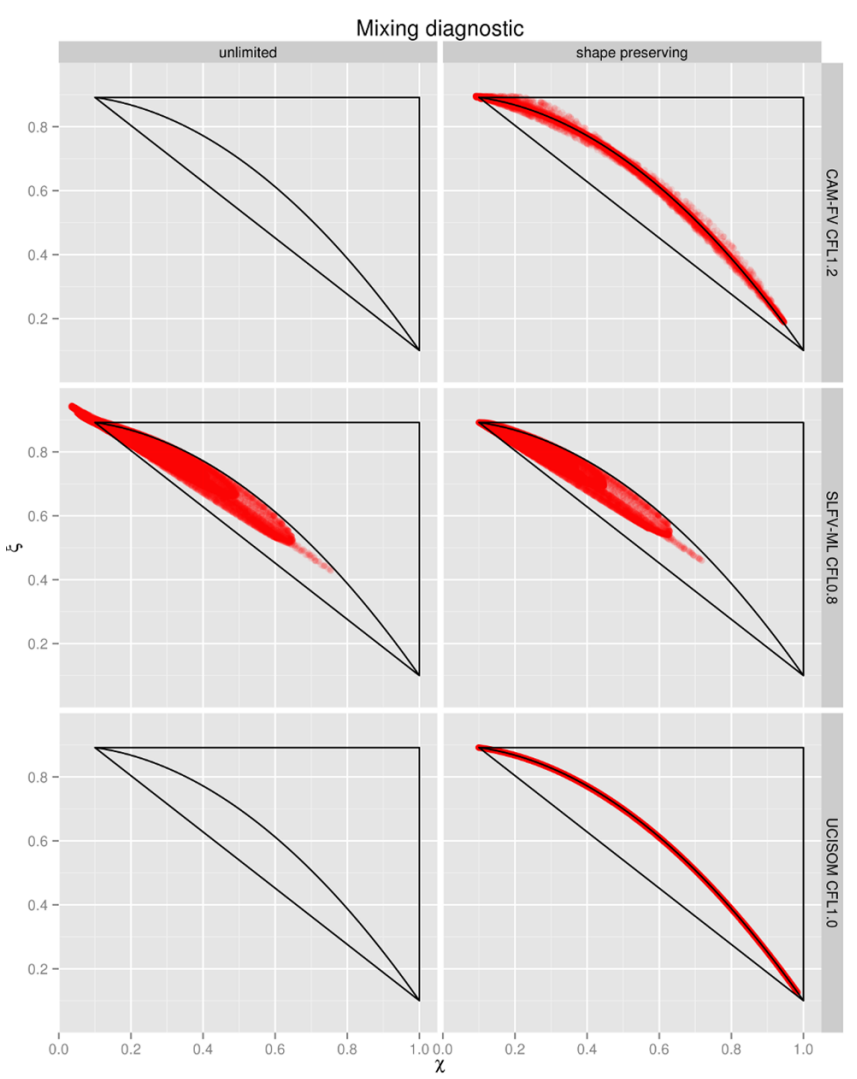

Figure 13. Mixing plots for both unlimited and shape-preserving versions. The resolution is set at $0.75^{\circ}$ at the Equator, except for Pangolin, which has $0.376^{\circ}$.

\section{Parallelization}

For large-scale numerical simulations, using only sequential computations is no longer affordable. To use a parallel approach, we need to split and balance the computational effort among a set of computing units. For partial different equation-based simulations where the computational cost is evenly shared among the cells, a natural and widely used approach is to partition the computational domain into connex subdomains of similar sizes. Each subdomain is handled by a different computing unit that leads a well-balanced parallel calculation.

The original objectives in designing this model were twofold. First, we intended to have a discretization with cells of equal areas so that the CFL condition is not penalized by the smallest cells. Second, we targeted a semi-structured grid to avoid managing complex data structures, as well as an extra-tool to generate it. This leads us to define the grid detailed in Sect. 2.2, where computing the neighbors of grid cells is fully algebraic. In a parallel framework, this grid has an additional asset as it enables a custom algebraic partitioning. Otherwise, mesh splitting often requires sophisticated mesh partitioning tools such as those developed by Karypis and Kumar (1995) and Pellegrini (2012). 

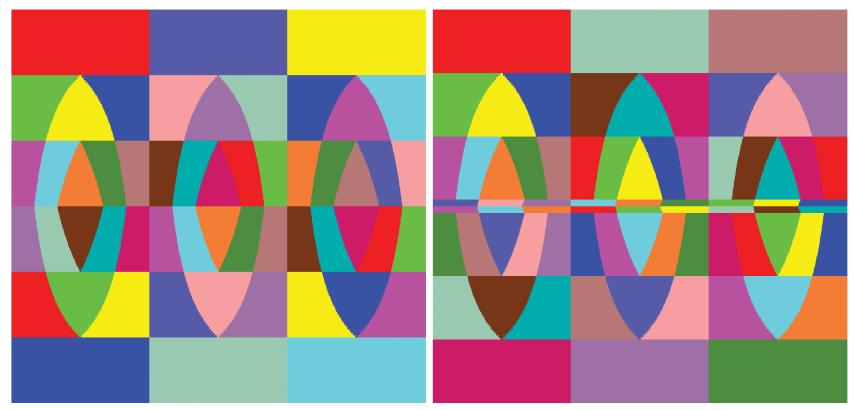

Figure 14. Algebraic mesh partitioning for an optimal case (54 domains, left) and sub-optimal case (74 partitions, left). Each color corresponds to a subdomain (the same color can be used for different subdomains). The grid contains 48600 cells and is shown in latitude-longitude coordinates.

\subsection{Partitioning}

In order to perform the partitioning, we first exploit the grid symmetries. The grid is composed of six identical zones as described in Sect. 2.2. We then focus on partitioning one of these zones, which contains $n_{\text {lat }}^{2}$ cells, where $n_{\text {lat }}$ is the number of latitudes in the hemisphere. A perfect work balance occurs when there are $p^{2}$ subdomains with $p$ dividing $n_{\text {lat }}$. In this case, each subdomain contains exactly $\left(n_{\text {lat }} / p\right)^{2}$ cells. The most natural way to gather cells to form the subdomains is to use the same algorithm as the one to build the grid. The $p^{2}$ subdomains are set on $p$ bands, where the $k$ th band contains $2 k-1$ subdomains $(k>0)$. Applying the same decomposition to the remaining five zones leads to the structure shown in Fig. 14.

For the sake of flexibility, this optimal situation can be slightly degenerated to accommodate any number of subdomains. For these sub-optimal situations, we consider the closest lower square $p^{\prime 2}$ on a zone, with $p^{\prime}$ dividing $n_{\text {lat }}$. These $p^{\prime 2}$ subdomains are set according to the previous strategy. The remaining $p^{2}-p^{\prime 2}$ cores are associated with on a special band, with less cells and thus without preserving the partition size.

These results are given for one zone only. For the complete grid, we find the optimal number of subdomains is $6 p^{2}$ with $p$ dividing $n_{\text {lat }}$. Otherwise, the model can manage any number of subdomains on one-third of the grid (between longitude 0 and 120). The total number of subdomains in these optimal cases is then a multiple of 3 . It is worth noting that White and Dongarra (2011) need a condition similar to our perfect case for their parallel version: the number of subdomains must be of the form $6 p^{2}$, with $p$ dividing the number of cells on a cube edge.

This algebraic partitioning uses the regular topology of the grid to create subdomains with a regular shape. This feature ensures regular data access and allows for possible optimizations by anticipation strategies such as pre-fetching to im-
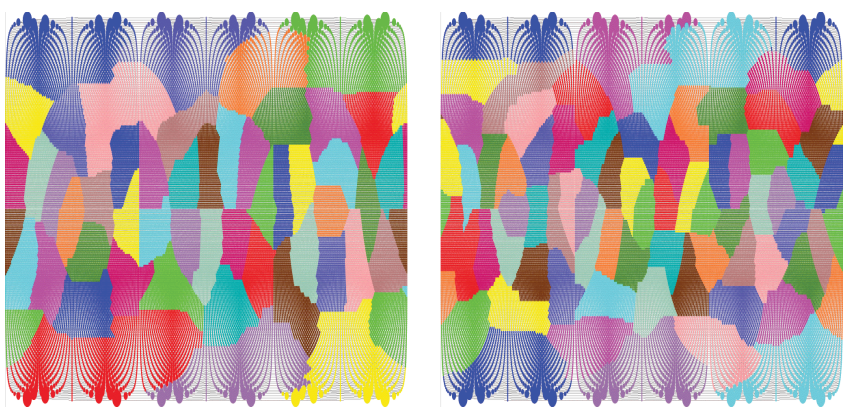

Figure 15. Mesh partitioning computed by the general purpose mesh partitioner Scotch for 54 domains (optimal case for Pangolin, left) and 72 domains (sub-optimal case for Pangolin, right). Each color corresponds to a subdomain (the same color can be used for different partitions). The grid contains 48600 cells and is shown in latitude-longitude coordinates.

prove faster data access by loading data into the cache before it is actually needed. To highlight this regularity, and for the sake of comparison, we display in Fig. 15 the partition computed by the mesh partitioner Scotch (Pellegrini, 2012) for the same grid as in Fig. 14. One can observe that this general-purpose tool does not succeed in preserving the regular shape of the subdomains. In addition, our partitioning reduces the number of neighbors for the subdomains and consequently the number of messages exchanged. The total number of neighbors of our partitioning is divided by at least 2 when compared with Scotch, even in sub-optimal cases.

\subsection{Parallel implementation}

Our parallel implementation first targets distributed memory architectures. Therefore, we consider a message passing parallel implementation on top of the MPI library where each subdomain is assigned to a different computing unit. To update the tracer ratio for all the cells in a subdomain, most of the information required to compute the fluxes is already available in the subdomain. However, some communications need to be performed to exchange information along the interfaces generated by the partitioning. For example, zonal fluxes need to exchange concentration and gradient data with the east and west neighboring subdomains. In that respect, we introduce ghost cells which store data received from the neighbors via message exchanges. It should be noted that, due to the shape of the subdomains, meridional advection requires communications with the north, south, east and west neighbors. This is illustrated in Fig. 16, where the ghost cells are shown as hatched cells.

In order to improve the parallel performance of the code and hide the communication time as much as possible, nonblocking communications are used. This avoids waiting for the completion of communications and allows for computations to be optimized. In practice, we first post the communication requests, and then perform the calculation on the 


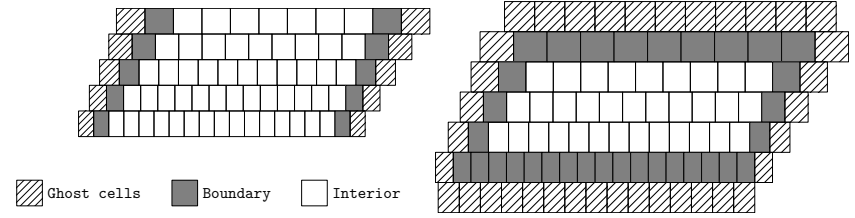

Figure 16. Ghost, boundary and interior cells for zonal (left) and meridional (right) advection.

interior cells, which do not need data outside the current subdomain. Then we finalize data reception and eventually compute the new quantities on the boundary cells using the values received in the ghost cells. This approach is a rather classical implementation to overlap computation and communication in large-scale simulations. The specificity of Pangolin comes rather from the algebraic features of the decomposition, so the neighbors of a subdomain and the cells inside it are computed on the fly. The model was also designed to have only one layer of ghost-cells as to decrease the communication volume.

Each advection step can be decomposed into several tasks: gradient computing, fluxes computing and mass update. The first two tasks require some communication and the nonblocking approach is used for each one of them. It should be noted that meridional advection requires an extra communication as it needs a zonal gradient to perform an interpolation (see Sect. 2.3). The different tasks are shown in detail in the algorithms shown in Fig. 17. The combination of boundary and interior cells for all these tasks is shown in Fig. 16.

The boundary zone is much larger for meridional advection as data need to be exchanged with the zone's four neighbors. More precisely, computation of the zonal gradient requires communication with the east and west neighbors of the subdomain. Furthermore, computing the meridional gradient and fluxes requires access to the north and south neighbors. Due to the semi-structured layout of the grid, this results in the "stair-like" structure for boundary cells in the meridional advection.

Most of the computation is performed during meridional advection. First, due to the extra step of computing the zonal gradient, meridional advection requires three message exchanges (vs. two for zonal advection). Also, the number of boundary cells is larger, thus increasing the communication volume. Finally, computation in a cell is more expensive as the number of neighbors is four on average (vs. two in the zonal case).

\subsection{Performances}

In this section, we investigate the parallel scalability of our implementation of the numerical scheme. Tests were done on the Bull cluster at CERFACS, whose features are shown in Table 3. We consider a strong speedup study where the size of the global grid is fixed and the number of computing
Table 3. Configuration for one of the 158 Sandy Bridge nodes.

$$
\begin{aligned}
& \text { - } 2 \text { Intel Sandy Bridge } 8 \text {-core CPUs } \\
& \text { (peak performance of } 330 \text { GFLOPS s} \\
& -32 \text { GB of memory } \\
& -31 \mathrm{~KB} \mathrm{~L} 1 \text { cache, } 256 \mathrm{~KB} \text { L } 2 \text { cache for each core } \\
& -20 \mathrm{MB} \text { L3 cache, shared by the } 8 \text { cores of each CPU }
\end{aligned}
$$

cores is increased. Ideally, the parallel elapsed time should be reduced proportionally to the number of cores selected for the parallel simulation. For our experimental study, we consider the elapsed time on three cores as the reference time so that the speedup is defined by

$S(p)=\frac{T(3)}{T(p)}$,

where $T(p)$ is the parallel elapsed time observed when performing the calculation on $p$ cores.

In strong speedup studies, the number of cells per subdomain decreases when the number of subdomains increases. Even though we attempt to overlap the communication and the calculation, communication volume tends to grow when the number of subdomains increases. A direct consequence is that the observed speedups gradually depart from the ideal speedup when the number of cores increases, as can be observed in Fig. 18 (left-hand side). On the right plot, we report experiments for the 2-D advection scheme using various grid sizes ranging from $1.13^{\circ} \times 0.75^{\circ}$ to $0.28^{\circ} \times 0.19^{\circ}$ when the number of cores varies from 3 to 128 . As expected, the larger the grid, the better the parallel performance, since we can better overlap calculation and computation. The speedup curves exhibit steps, with significant gaps when an optimal number of cores $(6,24,54,96)$ is used. In between, using more cores does not translate into an improvement in performance as the workload of the largest subdomain is not reduced.

The final version of Pangolin will be combined with chemistry modeling. As the chemistry computation is fully local, we can estimate the performances of the chemistryadvection model. Figure 18 (right-hand side) shows the estimated speedups of the complete chemistry advection simulation on the finest grid (i.e., $0.28^{\circ} \times 0.19^{\circ}$ ). We assumed the chemistry cost was constant across all cells and the chemical time step was similar to the advection time step. These assumptions are not valid in practice and only give an upper bound on the speedup. Nevertheless they give some insight into the final performances. The chemical time step was obtained from a new solver developed by D. Cariolle (personal communication, 2014) called ASIS with 90 species. As a reference, we use its implementation by P. Moinat, using the GMRES method (personal communication, 2014). As a result, adding the chemistry greatly increases the computational load in a subdomain and thus improves the scalability. On the other hand, communication volumes only increase linearly as a function of the number of tracers, as expected. 


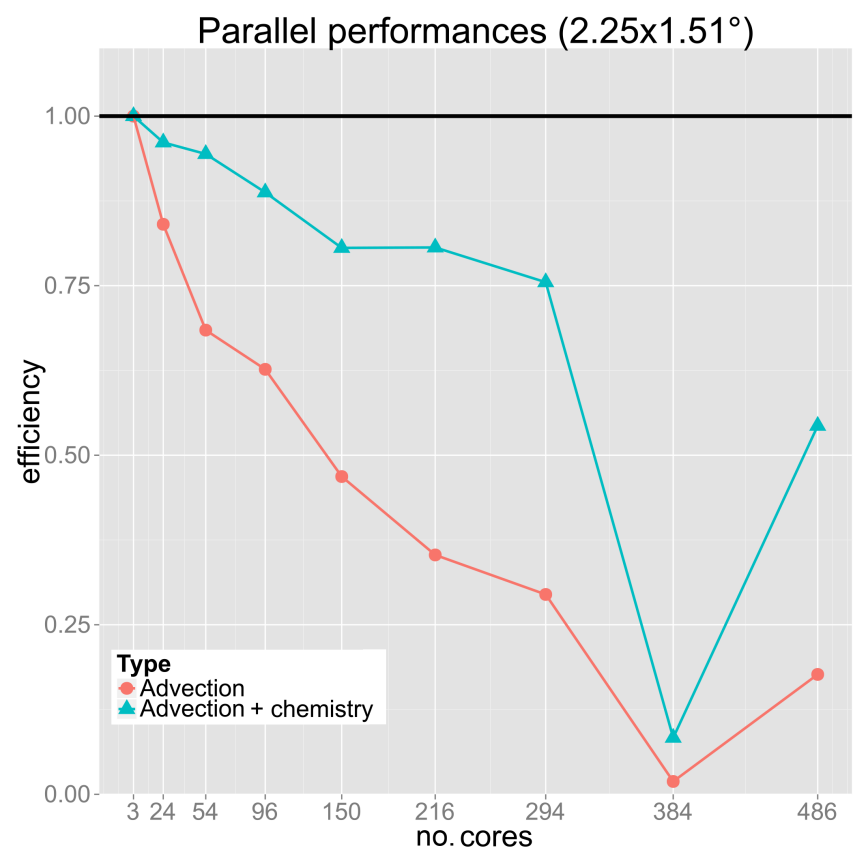

Figure 19. Efficiency up to 486 cores. A resolution of $2.25 \times 1.51^{\circ}$ was used to examine the limit of the parallelization performances for 2-D advection. For this test, the Airain cluster was used, which has 9504 nodes where each node has an Intel Xeon processor (16 cores, $2.7 \mathrm{GHz}$ and $4 \mathrm{~GB}$ of shared memory).

Table 4. Estimation of the number of cores needed for an efficiency of 0.75 for $2-D$ advection at several resolutions (lat $\times$ long).

\begin{tabular}{cc}
\hline Resolution & No. cores \\
\hline $1.5 \times 1.0$ & 150 \\
$0.75 \times 0.5$ & 486 \\
$0.15 \times 0.1$ & 6144 \\
\hline
\end{tabular}

sue, and a finite-volume formulation that ensures local conservation of the tracer mass. Grid features were carefully exploited to minimize memory requirements on the one hand, and provide maximal efficiency on parallel architectures on the other. The accuracy of the scheme was also chosen as to minimize the impact on message passing. It was found that the approximations made for computing the meridional gradients near the poles limits the accuracy of the model. Therefore, to reach the accuracy of other second-order models, resolution must be increased. This can be easily achieved without large computation penalty due to the good scalability of Pangolin.

We expect further improvement in terms of parallelism when chemistry is added. An ongoing work addresses realcase atmospheric situations using a linear scheme (Cariolle and Teyssèdre, 2007), which is used to model the evolution of stratospheric ozone on an isentropic surface. In future versions, vertical advection will be added, requiring a more ad-
Table 5. Some Pangolin configurations, with the number of latitudes on a hemisphere $n_{\text {lat }}$, total number of cells $n_{\text {pangolin }}$ and resolution at the Equator in degrees.

\begin{tabular}{rrc}
\hline$n_{\text {lat }}$ & $n_{\text {pangolin }}$ & $\Delta \phi \times \Delta \lambda$ \\
\hline 20 & 2400 & $4.5 \times 3.08$ \\
90 & 48600 & $1.0 \times 0.67$ \\
320 & 614400 & $0.28 \times 0.188$ \\
\hline
\end{tabular}

vanced correction of the winds for mass preservation. A complex chemistry will also be added using the ASIS solver and the RACMOBUS scheme (Dufour et al., 2005). This chemistry will most likely perturb the load balancing. One mitigation strategy would be to use multi-threading in the subdomains. To conclude, Pangolin is a practical model that is aimed at taking advantage of present and future parallel architectures for large-scale atmospheric transport.

\section{Code availability}

The code is copyright of the CERFACS laboratory. The documentation is available as a user manual and as code documentation at http://cerfacs.fr/ praga/pangolin/index.html. To request access to either the source code or documentation, please email A. Praga (alexis.praga@gmail.com) or D. Cariolle (cariolle@cerfacs.fr). The data and scripts for the plots of this paper are also available as a supplement.

\section{The Supplement related to this article is available online at doi:10.5194/gmd-8-205-2015-supplement.}

Acknowledgements. Financial support from the DGAC (Direction Générale de l'Aviation Civile) through the project IMPACT is gratefully acknowledged. A. Praga was supported by a PhD grant from Météo-France.

Edited by: A. Colette

\section{References}

Arakawa, A. and Lamb, V. R.: Computational design of the basic dynamical processes of the UCLA general circulation model, Meth. Comput. Phys., 17, 173-265, 1977.

Belikov, D., Maksyutov, S., Miyasaka, T., Saeki, T., Zhuravlev, R., and Kiryushov, B.: Mass-conserving tracer transport modelling on a reduced latitude-longitude grid with NIES-TM, Geosci. Model Dev., 4, 207-222, doi:10.5194/gmd-4-207-2011, 2011.

Cariolle, D. and Teyssèdre, H.: A revised linear ozone photochemistry parameterization for use in transport and general circulation models: multi-annual simulations, Atmos. Chem. Phys., 7, 2183 2196, doi:10.5194/acp-7-2183-2007, 2007. 
Chipperfield, M. P.: New version of the TOMCAT/SLIMCAT offline chemical transport model: Intercomparison of stratospheric tracer experiments, Q. J. Roy. Meteorol. Soc., 132, 1179-1203, doi:10.1256/qj.05.51, 2006.

Collins, W. and Rasch, P. J.: Description of the NCAR community atmosphere model (CAM 3.0), NCAR Tech. Note, 2004.

Dennis, J. M., Edwards, J., and Evans, K.: CAM-SE: A scalable spectral element dynamical core for the Community Atmosphere Model, Int. J. High Performance Comput. Appl., 26, 74-89, doi:10.1177/1094342011428142, 2011.

Dufour, A., Amodei, M., Ancellet, G., and Peuch, V.-H.: Observed and modelled "chemical weather" during ESCOMPTE, Atmos. Res., 74, 161-189, doi:10.1016/j.atmosres.2004.04.013, 2005.

Erath, C. and Nair, R. D.: A conservative multi-tracer transport scheme for spectral-element spherical grids, J. Comput. Phys., 271, 244-260, doi:10.1016/j.jcp.2014.04.008, 2014.

Godunov, S. K., Zabrodin, A. V., and Prokopov, G. P.: A computational scheme for two-dimensional nonstationary problems of gas dynamics and calculation of the flow from a shock wave approaching a stationary state, Zhurnal Vychislitel'noi, 1, 10201050, 1961.

Guba, O., Taylor, M., and St-Cyr, A.: Optimization-based limiters for the spectral element method, J. Comput. Phys., 267, 176-195, doi:10.1016/j.jcp.2014.02.029, 2014.

Hourdin, F. and Armengaud, A.: The use of finite-volume methods for atmospheric advection of trace species. Part I: Test of various formulations in a general circulation model, Mon. Weather Rev., 127, 822-837, 1999.

Huijnen, V., Williams, J., van Weele, M., van Noije, T., Krol, M., Dentener, F., Segers, A., Houweling, S., Peters, W., de Laat, J., Boersma, F., Bergamaschi, P., van Velthoven, P., Le Sager, P., Eskes, H., Alkemade, F., Scheele, R., Nédélec, P., and Pätz, H.-W.: The global chemistry transport model TM5: description and evaluation of the tropospheric chemistry version 3.0, Geosci. Model Dev., 3, 445-473, doi:10.5194/gmd-3-445-2010, 2010.

Jöckel, P., von Kuhlmann, R., Lawrence, M. G., Steil, B., Brenninkmeijer, C. A. M., Crutzen, P. J., Rasch, P. J., and Eaton, B.: On a fundamental problem in implementing flux-form advection schemes for tracer transport in 3-dimensional general circulation and chemistry transport models, Q. J. Roy. Meteorol. Soc., 127, 1035-1052, doi:10.1002/qj.49712757318, 2001.

Karypis, G. and Kumar, V.: Metis-unstructured graph partitioning and sparse matrix ordering system, version 2.0, Citeseer, available at: http://citeseerx.ist.psu.edu/viewdoc/summary?doi=10.1. 1.38.376 (last access: 4 February 2015), 1995.

Lauritzen, P. H., Skamarock, W. C., Prather, M. J., and Taylor, M. A.: A standard test case suite for two-dimensional linear transport on the sphere, Geosci. Model Dev., 5, 887-901, doi:10.5194/gmd-5-887-2012, 2012.
Lauritzen, P. H., Ullrich, P. A., Jablonowski, C., Bosler, P. A., Calhoun, D., Conley, A. J., Enomoto, T., Dong, L., Dubey, S., Guba, O., Hansen, A. B., Kaas, E., Kent, J., Lamarque, J.-F., Prather, M. J., Reinert, D., Shashkin, V. V., Skamarock, W. C., Sørensen, B., Taylor, M. A., and Tolstykh, M. A.: A standard test case suite for two-dimensional linear transport on the sphere: results from a collection of state-of-the-art schemes, Geosci. Model Dev., 7, 105-145, doi:10.5194/gmd-7-105-2014, 2014.

LeVeque, R. J.: Finite volume methods for hyperbolic problems, Cambridge University Press, 2002.

Machenhauer, B., Kaas, E., and Lauritzen, P. H.: Finite volume methods in meteorology, Methods Atmos., M, 3-120, doi:10.1016/S1570-8659(08)00201-9, 2009.

Miura, H.: An Upwind-Biased Conservative Advection Scheme for Spherical Hexagonal-Pentagonal Grids, Mon. Weather Rev., 135, 4038-4044, doi:10.1175/2007MWR2101.1, 2007.

Pellegrini, F.: PT-Scotch and LibPTScotch 0.6 User's Guide, Tech. rep., INRIA Bordeaux Sud-Ouest, 2012.

Prather, M. J.: Numerical advection by conservation of secondorder moments, J. Geophys. Res., 91, 6671-6681, 1986.

Rood, R. B.: Numerical advection algorithms and their role in atmospheric transport and chemistry models, Rev. Geophys., 25, 71-100, 1987.

Staniforth, A. and Thuburn, J.: Horizontal grids for global weather and climate prediction models: a review, Q. J. Roy. Meteorol. Soc., 138, 1-26, doi:10.1002/qj.958, 2012.

Teyssèdre, H., Michou, M., Clark, H. L., Josse, B., Karcher, F., Olivié, D., Peuch, V.-H., Saint-Martin, D., Cariolle, D., Attié, J.-L., Nédélec, P., Ricaud, P., Thouret, V., van der A, R. J., VolzThomas, A., and Chéroux, F.: A new tropospheric and stratospheric Chemistry and Transport Model MOCAGE-Climat for multi-year studies: evaluation of the present-day climatology and sensitivity to surface processes, Atmos. Chem. Phys., 7, 58155860, doi:10.5194/acp-7-5815-2007, 2007.

van Leer, B.: Towards the ultimate conservative difference scheme. IV. A new approach to numerical convection, J. Comput. Phys., 23, 276-299, 1977.

White, J. B. and Dongarra, J. J.: High-performance high-resolution semi-Lagrangian tracer transport on a sphere, J. Comput. Phys., 230, 6778-6799, doi:10.1016/j.jcp.2011.05.008, 2011.

Williamson, D. L.: The evolution of dynamical cores for global atmospheric models, Meteorol. Soc. Jpn., 85, 241-269, 2007.

Williamson, D. L., Drake, J. B., Hack, J. J., Jakob, R., and Swarztrauber, P. N.: A standard test set for numerical approximations to the shallow water equations in spherical geometry, J. Comput. Phys., 102, 211-224, 1992. 